Meta

Journal des tradlucteurs

Translators' Journal

\title{
EACROTANAL : Séminaire de terminologie, Zanzibar, 4-17 décembre 1983
}

\section{Jean-Jacques Lafitte Houssat, André Clas et Jean Baudot}

Volume 29, numéro 3, septembre 1984

URI : https://id.erudit.org/iderudit/002288ar

DOI : https://doi.org/10.7202/002288ar

Aller au sommaire du numéro

\section{Éditeur(s)}

Les Presses de l'Université de Montréal

\section{ISSN}

0026-0452 (imprimé)

1492-1421 (numérique)

Découvrir la revue

\section{Citer cette note}

Lafitte Houssat, J.-J., Clas, A. \& Baudot, J. (1984). EACROTANAL : Séminaire de terminologie, Zanzibar, 4-17 décembre 1983. Meta, 29(3), 333-334.

https://doi.org/10.7202/002288ar d'utilisation que vous pouvez consulter en ligne. 
EACROTANAL :

SEMINAIRE DE TERMINOLOGIE, ZANZIBAR, 4-17 DÉCEMBRE 1983

Pour donner suite aux divers vœux exprimés par les participants des diverses réunions et confé-

13. Donald Buchanan (1963) : dans Journal des Traducteurs, Montréal, vol. $8, n^{\circ} 4$, octobre-décembre, pp. 141-144, p. 142. Donner au lecteur "le sentiment qu'il lit une ceuvre originale ", René Aubenas, dans $l a$ Qualité en matière de traduction, Bad Godesberg, op. cit., p. 62, et Claude Elsen, ibid., p. 74. 
rences (Bujumbura, 1976 ; Bamako, 1979 ; Dakar, 1980 ; Yaoundé, 1983) sur la revalorisation et l'intégration des langues nationales dans les systèmes éducatifs, l'Eastern African Centre for Research on Oral Tradition and African National Languages (EACROTANAL) a organisé du 4 au 17 décembre 1983 un séminaire international de terminologie. L'EACROTANAL, organisation internationale regroupant la Tanzanie, le Burundi, les Comores, l'Éthiopie, Madagascar, la Somalie et le Soudan, a réuni les délégués de la plupart de ces pays pour participer au séminaire de formation en terminologie. $M$. Henri Rahaingoson, directeur de la recherche et des publications, EACROTANAL, a pu organiser cette rencontre grâce aux appuis financiers de l'UNESCO, de SIDA (Suède) et de l'ACDI (Canada). Les cours de terminologie et de terminotique étaient assurés par les soussignés, l'école de Wüster et INFOTERM ont été présentés par C. Galinski, les travaux de créativité terminologique en amharique ont été exposés par Amsalu Aklilu (université d'Addis Abeba), la recherche terminologique en luanda par $\mathrm{L}$. Walusimbi (université de Makerere, Ouganda).

\section{l'expérience éthiopienne}

On sait qu'il existe en Éthiopie (32 millions d'habitants) environ 90 langues, appartenant aux groupes sémitique, cushitique et nilotique. L'amharique est la première langue officielle (création en 1972 de la "National Amharic Academy "), la langue maternelle d'environ $20 \%$ de la population et la lingua franca d'un grand nombre d'Éthiopiens. L'Académie a entrepris un vaste projet de développement terminologique principalement dans les sciences naturelles, la technologie, l'agriculture et la nutrition. Là, comme ailleurs, il n'y a pas de règles immuables de création, mais le respect du principe qui veut que la créativité soit guidée par le système morphophonologique et syntaxique de la langue.

\section{l'expérience ougandaise}

L'Ouganda a environ 38 langues et l'anglais est langue officielle. La situation linguistique est confuse dans ce pays puisque le ministère de l'Éducation reconnaît six langues nationales d'enseignement, mais le ministère de l'Information (Radio) utilise plus de dix langues pour diffuser ses programmes d'enseignement et le ministère de la Culture et du Développement emploie plus de 20 langues dans ses campagnes d'alphabétisation. Même les organisations religieuses, utilisant la même langue, désignent la même réalité différemment. Ainsi les catholiques parlent de "Bibilia" et les protestants de "Bayibubi " pour désigner la Bible. Néanmoins, des efforts sont faits grâce à l'" Uganda Linguist and Authors Association " et à la "Luganda Language Society " pour la promotion et le développement des langues nationales. Signalons en passant la splendide création pour désigner la notion de littérature, soit ebiwandiiko ebiraga amagezi g'abantu, c'est-à-dire littéralement "l'écriture qui montre la sagesse du peuple».

On voit que toute l'Afrique est un laboratoire de recherches terminologiques orienté vers le développement et la promotion des langues nationales pour répondre aux nécessités du monde moderne.

ANDRÉ Clas ET JeAN BAUdot 